\title{
Influence of Coal Caving Time and Tail Beam Swing Angle on the Evolution of the Coal Gangue Boundary Line during Top Coal Caving
}

\author{
Qingliang Zeng $\mathbb{D},{ }^{1,2}$ Yanpeng Zhu, ${ }^{1}$ Yang Yang $\mathbb{D},{ }^{1} \mathrm{Zhe} \mathrm{Li}^{1}$ and Lirong Wan $\mathbb{D}^{1}$ \\ ${ }^{1}$ College of Mechanical and Electronic Engineering, Shandong University of Science and Technology, Qingdao 266590, China \\ ${ }^{2}$ College of Information Science and Engineering, Shandong Normal University, Jinan 250358, China \\ Correspondence should be addressed to Yang Yang; sdkdyangyang@126.com
}

Received 23 March 2021; Revised 22 December 2021; Accepted 24 December 2021; Published 3 February 2022

Academic Editor: Pengfei Wang

Copyright (c) 2022 Qingliang Zeng et al. This is an open access article distributed under the Creative Commons Attribution License, which permits unrestricted use, distribution, and reproduction in any medium, provided the original work is properly cited.

\begin{abstract}
In the process of top coal caving mining, the caving time and the tail beam swing angle have an important impact on the evolution of the coal gangue boundary, which determines the top coal recovery rate and gangue content rate. Therefore, it is necessary to study the influence of the caving time and the tail beam swing angle on the top coal caving effect. For this purpose, based on Hertz-Mindlin contact theory, this paper established a simulation model of top coal caving and carried out a coal caving simulation with different caving times and tail beam swing angles. In the process of coal caving, under a specific tail beam swing angle and different caving times, this work studied the evolution characteristics of the coal gangue boundary. A simulation model of coal caving with variable time is established, and the influence mechanism of time on the coal caving effect is analyzed. Then, the evolution characteristics of the boundary are studied under different tail beam swing angles and the same caving time. A simulation model of coal caving with variable angles is established, and the influence mechanism of the tail beam swing angle on the coal caving effect is analyzed. Finally, the effects of caving time and tail beam swing angle on coal gangue discharge are studied, and the effect of coal gangue release under different working conditions is analyzed. The boundary data and the discharge data are extracted through the simulation, and contrast curves are drawn. The results show a limit position of the coal gangue boundary under the fixed working condition, which is the middle line position of the coal caving outlet. When the coal gangue boundary does not reach the limit position, there are two characteristics. First, the greater the tail beam swing angle is, the faster the deformation speed of the coal gangue boundary is. At the same caving time, the position of the coal gangue boundary will be closer to the coal caving outlet, which leads to excessive mixing of coal and gangue. Second, the longer the drawing time, the greater the deformation of the coal gangue boundary, and the easier the coal gangue is mixed, resulting in an increase in the gangue content of the discharged coal. When the coal gangue boundary reaches the limit position, the boundary will be basically stable near the top of the coal caving outlet. The limit position and shape of the coal gangue boundary are mainly affected by the density difference of coal gangue particles but not by the coal caving time and the tail beam swing angle. After the end of coal caving, the coal gangue boundary finally presents a double arch structure. The research in this paper has important reference significance for the selection of caving time and tail beam swing angle and further research.
\end{abstract}

\section{Introduction}

In the mining of thick coal seams, fully mechanized caving mining is a mature and main technology that is widely used. To explore its mining methods and related laws, famous scholars in many countries have performed a large amount of targeted research, such as the United States, China, Poland, and so on [1]. The application of these studies has greatly improved the quality and efficiency of coal production and improved the intelligent automation and safety of coal mines. Using two-dimensional discrete element numerical simulation software PFC, Zhang et al. investigated 
the flow characteristics and loss rule of top coal in fully mechanized caving mining of extra-thick coal seams and obtained the original position, distribution, and migration rule of collapse top coal and lost coal [2]. Song et al. established a numerical model using Particle Flow Code software (PFC) that analyzed the top coal caving process and recovery ratio under different top coal thicknesses and obtained a reasonable top coal thickness in fully mechanized caving mining [3]. Wang et al., based on the BBR research system, optimized the caving mode of fully mechanized caving mining in extra-thick coal seams, proposed the subsection caving with a large interval, gave the determination formula of a reasonable caving interval under the condition of extra-thick top coal, and discussed the sensitivity of the interval on the thickness of broken top coal, the thickness of broken direct roof, and the eccentricity of loose body, which greatly improved the recovery rate of top coal [4]. Szurgacz and Brodny developed a new method of testing the position of the section in a longwall based on an analysis of its geometry. The method allows precise determination of the actual section settings over time. Its results can be used to analyze the work of the powered roof support operating in variable mining and geological conditions so as to optimize the support section. The results of the research play an important role in improving safety at work and the efficiency of the mining process [5]. According to different stages of mining, Zhang et al. carried out a theoretical analysis on the influencing factors of the mass ratio and proposed three measures to control the mass ratio [6]. Zhang et al. used field tests and numerical simulations to study the fracture development characteristics of overlying strata in the mining process of a fully mechanized mining face, which is of great significance for water disaster prevention and gas control of coal mines [7]. By using the finite-difference code called FLAC 3D, B. Unver and Yasitli conducted 3D modeling and numerical comparison simulation on the top coal lofting mechanism of a long wall panel and proposed an optimized prebursting blasting strategy, which can maintain the liquidity and continuity of coal caving behind the working face [8]. Zhang and $\mathrm{Wu}$ carried out research on the parameters and equipment configuration of a fully mechanized top coal caving face using numerical simulation. The results showed that enlarging the length of the fully mechanized caving face and cutting height had favorable effects on safe, efficient, and high-recovery mining [9]. According to GERRC technology, $\mathrm{Hu}$ et al. studied the impact of the shockproof barrier on reducing the impact of sliding coal gangue, which is significant for improving coal gangue protection technology [10]. By using the PFC2D calculation program, Zhang et al. analyzed the coal gangue flow field characteristics and top coal caving law under the conditions of different thicknesses of top coal and extraction-discharge ratios. This shows that the top coal loss is a process that gradually accumulates with the coal drawing cycle, and the velocity field and contact force field of coal gangue flow take the shape of a half arch centered on the coal drawing port; the smaller the distance from the coal caving outlet is, the smaller the contact force is [11]. Szurgacz and Brodny combine three areas: the forecasted load impacting the roof support, the tests covering selected systems and elements of the section, and the legal conditions regarding the admission of the roof support to operation. The main purpose of this was to develop a comprehensive methodology based on an analysis of safety and control systems, to test and assess the possibility of using powered roof support for given geological conditions. This methodology enables better adaptation of the support to given conditions. It also reduces the presence of miners in hazardous underground exploitation zones and improves efficiency. This will boost the sustainable development of the mining industry [12]. According to similarity theory, Liu et al. studied the flow and caving law of coal gangue and the top coal recovery rate in fully mechanized caving mining with a large mining height in a thick coal seam under different caving intervals, studied the influence law of mechanical mining height on top coal caving and mixed gangue, and determined reasonable caving technological parameters [13]. Yu et al. fitted the formula of extreme point shift of top coal subsidence curve by building a test model; also, they established the equation of the coal gangue boundary and top coal evolved body according to the random medium theory [14]. The results show that the limiting action of the tail beam and the mutation of caving conditions caused by the coal caving outlet have important influences on the coal gangue boundary and the shape of the top coal caving body. Through the combination of theoretical analysis with numerical simulation, Zhu et al. studied the dynamic evolution of the top coal caving body in fully mechanized caving coal mining [15]. They pointed out the limitations of traditional ellipsoid theory in analyzing the shape of the top coal caving body; the morphology and evolution law of the top coal caving body obtained by numerical simulation are consistent with the theoretical analysis results. This proves that it is feasible to apply stochastic medium theory to the analysis of the coal drawing process in fully mechanized caving mining. Zhang developed a 3D simulation test bench for loose top coal in fully mechanized caving mining. Using this test bench, they carried out a release test for loose top coal of fully mechanized top coal caving with upward and inclined mining [16]. This revealed the characteristics of the top coal recovery rate, top coal caving body, and coal gangue boundary under upward mining. At the same time, it provided a new test method for studying the top coal caving mechanism in fully mechanized top coal caving mining from a 3D perspective. According to the field industrial test of the intelligent fully mechanized caving working face, Zhang et al. realized the real-time monitoring and control of the size of the coal caving outlet in the whole process [17]. This provided the necessary conditions for realizing the intelligent unmanned mining of the fully mechanized caving working face. They studied the applicable sensing technology and equipment for different top coal caving stages and processes. Moreover, they monitored the process of top coal caving in real time, which provided the necessary means for realizing automatic intelligent caving of fully mechanized coal mining. Jia et al. carried out the theoretical analysis, numerical simulation, and field tests on a fully mechanized coal caving working face and analyzed the stress environment, deformation, and failure characteristics of a mining roadway on a fully 
mechanized caving working face. The results indicated that the asymmetric deformation of the floor slab of a mining tunnel is the stress asymmetry of the surrounding rock caused by mining [18]. Dai et al. studied the intelligent identification method for the stability of a cross section coal pillar and analyzed the load transfer law of the overlying strata in the upper part of the coal pillar. Through empirical formulas, a mean impact value-genetic algorithm-BP neural network (MIV-GA-BP) simulation experiment, and a finitedifference algorithm, each index of the coal pillar value simulation experiment was obtained [19]. Taking the Jinggong No. 3 mine 34201 working face of the Pingshuo Group as the research background, under different conditions, $\mathrm{He}$ et al. established numerical models of different bevel angles through PFC2D and analyzed the displacement field of the top coal, contact stress field, and incipient point of the top coal displacement. They inverted the shape of the top coal caving body and obtained the distribution law of space occupation of top coal loss. This provides a basis for determining reasonable parameters and for improving the recovery rate of top coal in on-site fully mechanized top coal caving mining [20]. Yang et al. studied the fracture characteristics of coal seam roofs and the sliding characteristics of caving gangue through theoretical modeling, bottom friction experiments, numerical calculations, and other methods. They showed the mechanism of coal wall failure under different coal seam occurrences and mining conditions and proposed specific measures to prevent coal wall failure and floor sliding [21]. Lan et al. obtained the stratum structure and stratum behavior of hard roof thick coal seam mining through field tests and proved the existence of a "high-rise structure" and its influence on strong stratum behavior [22].

The existing research has studied many aspects of fully mechanized top coal caving mining that are significant in improving fully mechanized caving mining technology. Fully mechanized caving mining is a research direction composed of a substantial theoretical system. However, there are still many research points that have not been deeply studied, such as the evolution of the coal gangue boundary. At present, there are few studies on the evolution of the coal gangue boundary. The evolution of the coal gangue boundary is not only an innovative research direction but also a research gap at present. It has a nonnegligible effect on the top coal caving process. The evolution of the coal gangue boundary directly affects the amount of top coal caving, the gangue mixing ratio, and the time of gangue collapse. It even has an important influence on the migration law of top coal and the destruction of overburden in fully mechanized caving mining [23].

The evolution of the coal gangue boundary is of great significance in improving the quality of top coal mining. Therefore, it is necessary to study it. The main task of this paper is to extract and record the evolution process of the coal gangue boundary by simulating top coal caving under different conditions. The main aim is to research the influence of the evolution of the coal gangue boundary on the top coal caving process by analyzing the evolution law of the coal gangue boundary.
In fully mechanized caving mining, there are many factors affecting the evolution trend of the coal gangue boundary. Under the specific coal-caving environment, the most important factors are the time of coal caving and the swing angle of the tail beam. The coal caving time determines the amount of coal and gangue particles flowing to the coal caving outlet above the hydraulic support, while the tail beam swing angle directly determines the size of the coal caving outlet. If the coal caving outlet is too large, the gangue content will be increased. In contrast, if the coal caving outlet is too small, the top coal drawing efficiency will be reduced. For a given working condition, from a technical point of view, there must be a reasonable drawing time and a reasonable swing angle of the tail beam. Thus, the top coal caving process has the optimal evolution of the coal gangue boundary. Thus, fully mechanized caving mining has the best economic benefits and takes into account the caving efficiency and coal quality. Therefore, based on Hertz-Mindlin contact theory, this paper simulates the evolution of the coal gangue boundary by the discrete element method under different drawing times and different tail beam pendulum angles. Taking the coal gangue boundary as the research object, the evolution data of the boundary are extracted, and the influence of its evolution on the top coal recovery rate and coal gangue mixing rate is analyzed.

The research objective of this paper is to find out the evolution law of the coal gangue boundary and then summarize the influence of drawing time and tail beam swing angle on its evolution. In the top coal caving process, the drawing time and tail beam swing angle have a significant influence on the flow of coal and gangue, and the flow has an important impact on the caving effect. Therefore, to improve the quality of top coal caving mining, it is very important and necessary to study the evolution law of the coal gangue boundary. The existing research on the evolution of the coal gangue boundary is still very few, so this paper will fill gaps in the research on it and make an important contribution to perfecting the theoretical system of top coal caving. The results obtained in this paper are of great significance to improve the quality of fully mechanized caving mining and promote the construction of the smart mine.

\section{Theoretical Basis of the Simulation Model of the Coal Caving Process}

At present, an effective method to study the flow of discrete particle groups is the discrete element simulation method [24]. The basic idea of discrete element simulation is as follows: under the action of external force, movement and power transfer between particles will inevitably occur, and the movement of particles is bound to cause contact and collision between particles, so there is also bound to be new displacement and stress between particles. Based on this idea, discrete element simulation divides the research object into a finite number of entity elements independent of each other, and the size can be customized. In the Newton coordinate system, according to the interaction between elements and the law of motion, iterative methods such as the 
dynamic relaxation method and static relaxation method are used for cyclic iterative calculation. In this way, the force and displacement of all elements in each time step are determined, and the position and stress state of all elements are updated constantly over time. Finally, by tracking and calculating the micromotion of each element, the overall macromotion or the granular flow law of the whole research object can be obtained [25].

In this study, spherical particles are used for coal gangue particles to facilitate the mechanical contact between particles [26] and simulation calculations. To compensate for the influence of the shape between particles on the simulation results, the coal and gangue particles were randomly changed in volume based on a set radius. The friction effect, bonding effect, and normal elastic-plastic between particles were added through a theoretical model. When the coal gangue particle group flows under stress, the contact surface between particles has significant friction, so the damping component of both the normal force and the tangential force should be considered simultaneously [27]. At present, Hertzian contact theory is a mature theory in the calculation of the normal force of particles [28]. Small deformation occurs in the normal contact area of coal and gangue particles, and the contact surface can be considered an ellipse. When coal gangue particles are regarded as elastic halfspaces in the normal direction, Hertzian contact theory is very applicable. In calculating the tangential force of particles, the established theory is the Mindlin-Deresiewicz contact theory [29]. On the contact surface of coal gangue particles, the increment of relative tangential displacement is related to the increment of tangential force between particles. Therefore, it can be considered that the particles have small plastic deformation or no plastic deformation under ideal conditions. Here, the Mindlin-Deresiewicz contact theory is applicable. There are two main kinds of contact calculations in discrete element simulation of the coal gangue boundary of caving coal: contact calculation between particles and support and contact calculation between particles. In this study, it is considered that the coal gangue particles and the hydraulic support are in rigid contact, and the normal support force and friction force of the support to the particles are mainly considered, so the Hertz-Mindlin (no-slip) contact model is adopted [30]. The tangential friction conforms to the basic Coulomb friction law, and rolling friction is achieved by a contact-independent directional constant torque model. That is, rolling friction is realized by adding an independent directional torque between the particles and the contact surface of the hydraulic support. On the other hand, in the calculation of particle-toparticle contact, the coal gangue particles have an obvious bonding effect in the top coal caving process [31]. Based on Hertzian contact theory and Mindlin-Deresiewicz contact theory, the influence of bonding on particle motion must also be considered. Therefore, the Hertz-Mindlin with Bonding contact model is adopted for the contact between particles. The Hertz-Mindlin with bonding contact model can be used to bond particles. It can simulate the bond between each contact surface or contact point of particles through theoretical calculation. The bond can withstand limited tangential and normal displacements and fracture when the maximum allowable stress is reached [32]. The Hertz-Mindlin with Bonding contact model is especially suitable for the simulation of top coal caving in this paper.

Based on the above discussion, the Hertz-Mindlin (noslip) contact model is adopted for coal gangue particles and hydraulic support, and the Hertz-Mindlin with bonding contact model is adopted for coal gangue particles and particles in this study. The model theory is described below.

2.1. Hertz-Mindlin (No-Slip) Contact Model. In the simulation of top coal caving, the outer radii of any two simulation units is $R_{1}$ and $R_{2}$, and the calculation formula of overlap amount $\alpha$ of any two simulation units is as follows:

$$
\alpha=R_{1}+R_{2}-\left|r_{1}-r_{2}\right|,
$$

where $r_{1}$ and $r$ are the external ball position vectors of the two simulation units.

The contact surface between particles is considered to be circular, and the contact radius $a$ is as follows:

$$
a=\sqrt{a R^{*}},
$$

where $R^{*}$ is the equivalent particle radius, which can be obtained from the following formula:

$$
\begin{aligned}
& \frac{1}{R^{*}}=\frac{1}{R_{1}}+\frac{1}{R_{2}}, \\
& F_{n}=\frac{4}{3} E^{*}\left(R^{*}\right) \frac{1}{2} \alpha \frac{3}{2}, \\
& \frac{1}{E^{*}}=\frac{1-v_{1}^{2}}{E_{1}}+\frac{1-v_{2}^{2}}{E_{2}},
\end{aligned}
$$

where $E^{*}$ is the equivalent elastic modulus and $E_{1}, v_{1}, E_{2}$, and $v_{2}$ are the elastic modulus and Poisson's ratio of particle 1 and particle 2, respectively.

The normal damping force $F_{n}^{d}$ can be obtained from the following formula:

$$
F_{n}^{d}=-2 \sqrt{\frac{5}{6}} \beta \sqrt{S_{n} m^{*}} v_{n}^{r e l},
$$

where $m^{*}$ is the equivalent mass, which can be obtained from the following formula:

$$
m^{*}=\frac{m_{1} m_{2}}{m_{1}+m_{2}} .
$$

Let the velocity of two particles before collision be $\mathrm{v}_{1}$ and $v_{2}$, respectively, and the normal unit vector of collision be $n$; then:

$$
n=\frac{r_{1}-r_{2}}{\left|r_{1}-r_{2}\right|}
$$

In equation (6), $v_{n}^{r e l}$ is the normal component value of the relative velocity, which can be obtained from the following equation: 


$$
v_{n}^{r e l}=\left(v_{1}-v_{2}\right) \cdot n .
$$

The coefficient $\beta$ and normal stiffness $S_{n}$ in equations (2)-(6) can be obtained from the following two equations:

$$
\begin{aligned}
& S_{n}=2 E^{*} \sqrt{R^{*} \alpha}, \\
& \beta=\frac{\ln e}{\sqrt{\ln ^{2} e+\pi^{2}}},
\end{aligned}
$$

where $e$ is the recovery coefficient.

The tangential force $F_{t}$ between particles can be obtained from the following equation:

$$
\begin{aligned}
G^{*} & =\frac{2-v_{1}^{2}}{G_{1}}+\frac{2-v_{2}^{2}}{G_{2}}, \\
S_{t} & =8 G^{*} \sqrt{R^{*} \alpha}, \\
F_{t} & =-S_{t} \delta,
\end{aligned}
$$

where $\delta$ is the tangential overlap, $S_{t}$ is the tangential stiffness, $G^{*}$ is the equivalent shear modulus, and $G_{1}$ and $G_{2}$ are the shear moduli of the two particles.

The tangential damping force $f_{t}$ between particles can be obtained from the following formula:

$$
f_{t}=-2 \sqrt{\frac{5}{6}} \beta \sqrt{S_{t} m^{*}} v_{t}^{r e l},
$$

where $v_{t}^{\text {rel }}$ is the tangential component of the relative velocity.

The tangential force between particles is limited by Coulomb friction $\mu_{r} F_{n}$, where $\mu_{r}$ is the static friction coefficient.

The rolling friction in the simulation is very important, which can be explained by the torque on the contact surface

$$
T_{i}=-\mu_{r} F_{n} R_{i} \omega_{i},
$$

where $\mu_{r}$ is the rolling friction coefficient, $R_{i}$ is the distance between the center of mass and the contact point, and $\omega_{i}$ is the unit angular velocity vector of the object at the contact point.

2.2. Hertz-Mindlin with Bonding Contact Model. In the top coal caving simulation experiment, the coal gangue particles are in the bonding state at the initial setting. With the breaking and flowing of the top coal, the bonds of some coal gangue particles will be destroyed gradually. Assuming that the bond of any two coal and gangue particles is destroyed at moment $t_{\alpha}$, and after moment $t_{\alpha}$, the interaction between the two coal and gangue particles will occur through a common Hertz-Mindlin contact model. Before moment $t_{\alpha}$, the two coal and gangue particles will interact via the Hertz-Mindlin with bonding contact model. To make the theory and simulation closer to the real situation, the bonding forces $F_{n}$ and $F_{t}$ and torques $T_{n}$ and $T_{t}$ between particles will be gradually adjusted with the time step under the bonding flow model.
The bonding forces $F_{n}$ and $F_{t}$ and the torques $T_{n}$ and $T_{t}$ are adjusted in the same way as in the following formula:

$$
\begin{gathered}
\delta F_{n}=-v_{n} S_{n} A \delta_{t}, \\
\delta F_{t}=-v_{t} S_{n} A \delta_{t}, \\
\delta T_{n}=-\omega_{n} S_{t} J \delta_{t}, \\
\delta T_{t}=-\omega_{t} S_{n} \frac{J}{2} \delta_{t},
\end{gathered}
$$

where

$$
\begin{aligned}
& J=\frac{1}{2 \pi R_{B}^{4}}, \\
& A=\pi R_{B}^{2} .
\end{aligned}
$$

In the equation, $R_{B}$ is the contact bonding radius, $S_{n}$ and $S_{t}$ are the normal and tangential stiffness of particles, $\delta_{t}$ is the time step, $v_{n}$ and $v_{t}$ are the normal and tangential velocities of particles, and $\omega_{n}$ and $\omega_{t}$ are the normal and tangential angular velocities.

When the normal stress and tangential stress of the contact surface exceed the set maximum strength, the bond is broken. Therefore, the maximum values of the normal and tangential stresses are as follows:

$$
\begin{gathered}
\sigma_{\max }<\frac{-F_{n}}{A}+\frac{2 T_{t}}{J} R_{B}, \\
\tau_{\max }<\frac{-F_{t}}{A}+\frac{2 T_{n}}{J} R_{B} .
\end{gathered}
$$

The above bonding forces and moments in the Hertz-Mindlin with Bonding Contact Model are applied based on the standard Hertz-Mindlin Contact Theory. The basic theory is the same as that of the Hertz-Mindlin (noslip) Contact Model, which will not be repeated here.

\section{Establishment of Simulation Model for Top Coal Caving Process}

To study the influence of different tail beam parameters on the top coal gangue boundary in the process of top coal caving, according to the field conditions and similarity theory, a simulation model of the top coal caving process, as shown in Figure 1, is established in EDEM 2018. In the model, the hydraulic support type is ZF5600/16.5/26, the selected mining height is $2.3 \mathrm{~m}$, and the geometric ratio of the model to the prototype is $1: 1$. In the initial state, the tail beam is parallel to the shield beam. The thickness of the top coal above the top beam is $3.45 \mathrm{~m}$, which is paved and compacted over the top beam, shield beam, and tail beam. The thickness of the gangue above the top coal is $1.45 \mathrm{~m}$, while the width of the gangue on the support and right side of the top coal is $2.47 \mathrm{~m}$, which are all paved and compacted. To facilitate mechanical contact and simulation calculations, spherical particles are used for coal and gangue particles considering the nonuniformity of particle size after gangue crushing. To make the physical 


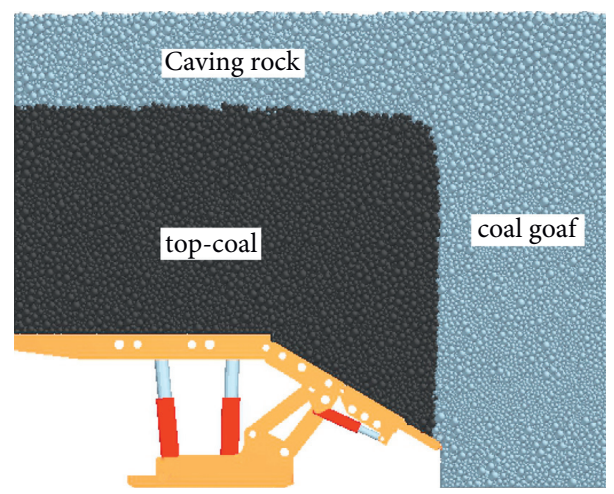

FIgURE 1: Simulation model of top coal caving process.

parameters of the simulation close to the actual situation as much as possible, the foundation diameter of coal gangue particles is set at $80 \mathrm{~mm}$ and randomly generated in the diameter range of 0.5 to 2 times. The specific material parameters [33] are shown in Table 1.

There are three main materials in the simulation experiment, namely, coal, gangue, and structural steel. It mainly involves the contact and collision between six materials, namely, the interaction between model materials: coal and coal, coal and gangue, coal and steel, gangue and gangue, gangue and steel, steel and steel. The simulation experiment only studies the influence of hydraulic support on coal gangue dumps, while the internal interaction of hydraulic support has no influence on coal gangue dumps. Therefore, the interaction between steel and steel is not considered. The interaction parameters of the other five kinds of contact are fundamental to the simulation experiment, mainly including the coefficient of restitution, static friction, and rolling friction between materials [34]. The specific parameters are shown in Table 2 .

To carry out a comparative study, we took the tail beam swing angle as the variable, set the variable range from $20^{\circ}$ to $40^{\circ}$, and set the variable interval between different simulations to $2^{\circ}$. Finally, 11 sets of simulation conditions are obtained. In the preparation stage of the simulation experiment, to obtain a more complete and accurate simulation model, this paper established the hydraulic support model in Solidworks 2017; then, the particle factory shell model required by gangue particles is generated using EDEM 2018. Then, the two models are accurately imported into the EDEM simulation environment through the rectangular coordinate system. In EDEM 2018, we developed top coal and caved rock through the particle factory model established in advance; also, we generated compacted coal and gangue using a solid particle factory model. The generation process of a large number of coal gangue particles is extremely slow, and the quantitative generation time has nothing to do with the subsequent simulation results. Therefore, this time is not included in the formal simulation time. The official simulation time starts with the moment when the tail beam begins to swing at $0 \mathrm{~s}$.

After the simulation starts, the coal caving outlet of the hydraulic support will gradually open, and the broken top coal will flow out slowly. At the same time, the collapse of the upper gangue and the rear accumulation of gangue will gradually close the coal caving outlet as the quantity increases. After a period of coal caving, a noticeable flow of the coal gangue boundary can be observed. At this point, the coal caving outlet will be gradually closed to finish a complete cycle of the swing of the tail beam, and the realtime position data of the coal gangue boundary line will be recorded and saved.

\section{Analysis of Simulation Results of Top Coal Caving Process}

In the process of top coal caving simulation, first, the tail beam swings down from the initial state to the specified angle. During this stage, the top coal falls at an accelerated rate, the coal gangue boundary moves from the initial static position to the lower left, and the deformation increases with time. It can be observed that with the enlargement of the coal caving outlet, the flow of the gangue particle group accelerates, and an obvious coal gangue boundary begins to appear. Then, the tail beam swings to the specified angle and stops swinging. During this stage, it can be observed that the flow of gangue particles is increasingly faster, the trend of gangue extrusion to the top coal crushing zone is increasingly larger, and the deformation of the coal gangue boundary is increasingly faster. After a period of coal caving, the coal gangue boundary curve presents an obvious arch shape. Finally, the tail beam swings up to its original position. During this stage, the coal caving outlet gradually decreases, and the flow speed of the top coal slows down. The coal gangue boundary continues to deform to the left and down, but the velocity of the deformation is increasingly slow. When the coal caving outlet is close to closing, the lower part of the coal gangue boundary line deforms toward the right gangue; that is, the lower part recovers a small deformation extent. Ultimately, the coal gangue boundary shows two reverse arches.

By extracting the above 11 groups of experimental results of the top coal caving simulation, the animation, images, and data of the coal gangue boundary under different working conditions are obtained. By using Origin 2018 to extract the coordinate information of the coal gangue boundary in the simulation image, the coal gangue boundary curve is finally generated. Here, taking the swing condition of the $30^{\circ}$ tail 
TABLE 1: Particle material parameters.

\begin{tabular}{lcccc}
\hline Material & Density $\left(\mathrm{kg} / \mathrm{m}^{3}\right)$ & Shear modulus $(\mathrm{pa})$ & Poisson ratio & Work function $(\mathrm{J})$ \\
\hline Coal & 800 & $\left(2.2 \times 10^{9}\right)$ & 0.28 & 0.3 \\
Gangue & 2900 & $\left(3.4 \times 10^{10}\right)$ & 0.3 & 0 \\
Steel & 7850 & $\left(2.06 \times 10^{11}\right)$ & 0.3 & \\
\hline
\end{tabular}

TABLE 2: Material interaction parameters.

\begin{tabular}{|c|c|c|c|c|c|}
\hline Interaction parameters & Coal-coal & Coal-gangue & Coal-steel & Gangue-gangue & Gangue-steel \\
\hline Coefficient of restitution & 0.64 & 0.645 & 0.65 & 0.645 & 0.6 \\
\hline Coefficient of static friction & 0.329 & 0.315 & 0.46 & 0.3 & 0.5 \\
\hline Coefficient of rolling friction & 0.036 & 0.028 & 0.032 & 0.02 & 0.01 \\
\hline
\end{tabular}

beam as an example, the simulation result image extracted in EDEM 2018 is shown in Figure 2. The coal gangue boundary curve extracted in Origin 2018 is shown in Figure 3.

\subsection{Influence of Caving Time on Change in the Coal Gangue} Boundary. To compare the influence of different caving times on the coal gangue boundaries, 9 time points with a span of 1 second were selected uniformly in the same tail beam swing cycle. The location data of the coal gangue boundary at the 9 time points in all experimental groups were extracted, and a total of 99 groups of data were obtained. Taking the $30^{\circ}$ working condition as an example, using the Origin 2018 digital chart tool, visual curves are generated, and 9 curves are summarized into the same coordinate system. A summary diagram of the coal gangue boundary is shown in Figure 4.

As shown in Figure 4, the difference in the coal gangue boundary in the first half is slight, and in the second half, it is obvious. For the convenience of research and display, the initial scale value of the horizontal axis of the coordinate system is set to 5.0 in the curve. This enlarges the major change details of the coal gangue boundary, as shown in Figure 5(a). Similarly, the other 10 groups of the coal gangue boundary simulation data were extracted, and a total of 11 coal gangue boundary curves at different times were obtained, as shown in Figure 5.

From a single curve, taking an average angle of $30^{\circ}$ as an example, it can be observed that from time 1 to time 2 , the flow trend of the coal gangue boundary curve is very small, and only the coal particles closest to the coal caving outlet have a large displacement. From time 2 to time 4, the flow trend of the curve began to increase. With the reduction in the distance between coal gangue particles and the coal caving outlet, the flow trend became increasingly larger. From time 5 to time 7 , the flow trend of the curve becomes more obvious, and the particle displacement and the flow velocity are larger. From time 8 to time 9 , the flow velocity of some particles is too high. The coal gangue boundary curve is no longer smooth, and local bending occurs, indicating that the mixed layer of coal gangue particles appears.

From comparing 11 groups of curves, under the specific swing angle condition, when the tail beam swings downward, the coal caving outlet gradually increases. Meanwhile, the coal gangue boundary gradually accelerates to the lower left, and the arched parabola becomes increasingly obvious. When the tail beam swings to the specified angle, the deformation speed of the coal gangue boundary down to the left is the fastest. When the tail beam swings upwards, the deformation of the coal gangue boundary continues to increase. Until near the 7 th second of simulated coal caving, the deformation of the boundary reaches the maximum value within one swing period of the tail beam, and the tail beam is approximately $1 / 2$ in the upward swing process. As the simulation continues, the tail beam continues to swing upward, and the coal caving outlet continues to decrease. It can be observed that the upper part of the coal gangue boundary is still in downward deformation, but the deformation velocity slows down, and the deformation change is very small. Meanwhile, the lower part of the boundary begins to return deformation and reverse deformation to the right. Because the lower part of coal gangue particles is close to the coal caving outlet, it is greatly affected by the swing of the tail beam, and the deformation is complex. This is manifested as follows: The boundary above $1.6 \mathrm{~m}$ of longitudinal coal depth deforms to the right, but the deformation is limited, and the shape of the arch section is basically stable. The boundary under $1.6 \mathrm{~m}$ deforms to the right, the deformation gradually increases with decreasing distance from the coal caving outlet, and the deformation is positively proportional to time.

4.2. Influence of Different Caving Angles on the Change in the Coal Gangue Boundary. To compare the influence of different tail beam swing angles on the coal gangue boundary, we evenly select 4 time points in the same tail beam swing period, which are $3 \mathrm{~s}, 5 \mathrm{~s}, 7 \mathrm{~s}$, and $9 \mathrm{~s}$. Then, 11 groups of coal gangue boundary position data at the selected time points are extracted, and a total of 44 groups of data are obtained. The 44 groups of data were summarized according to the 4 time points to generate a visual chart, and the horizontal axis initial value of the graph was set to 5.0. A total of 4 curves of the coal gangue boundary with different angles were obtained, as shown in Figure 6.

From the single curve view, when the coal caving time is $3 \mathrm{~s}$, the coal gangue boundary of all angles from $20^{\circ}$ to $40^{\circ}$ is smooth, the fluctuation is small, the deformation extent is 


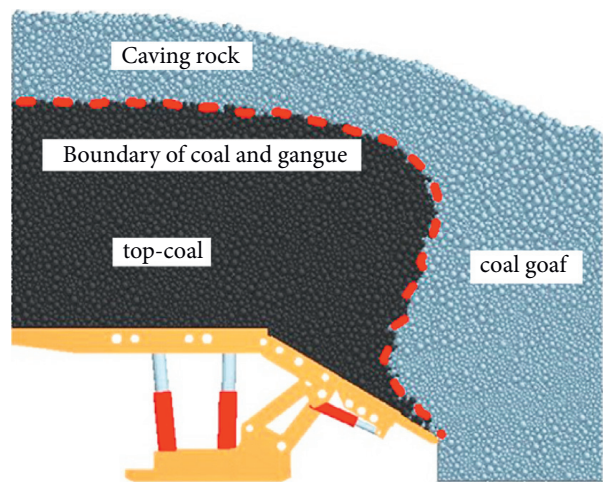

Figure 2: Result image of the coal gangue boundary.

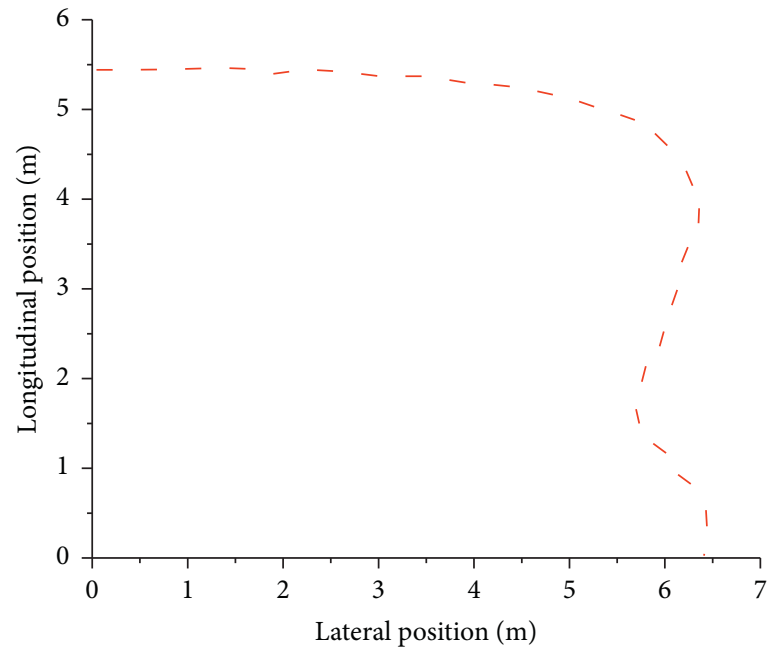

Figure 3: Curve of the coal gangue boundary.

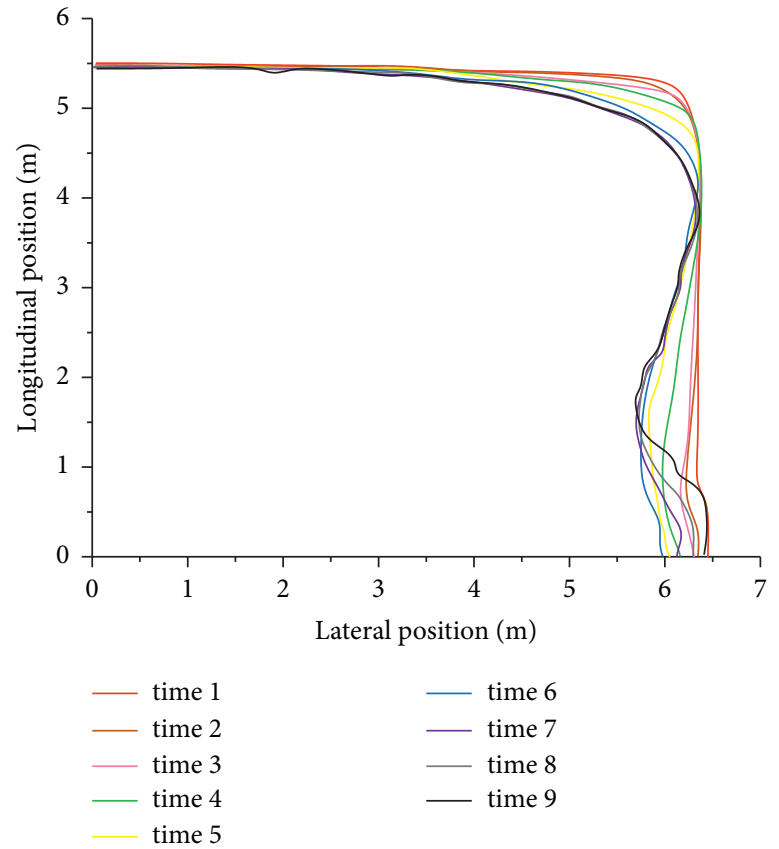

FIgURE 4: Summary diagram of the coal gangue boundary at $30^{\circ}$. 

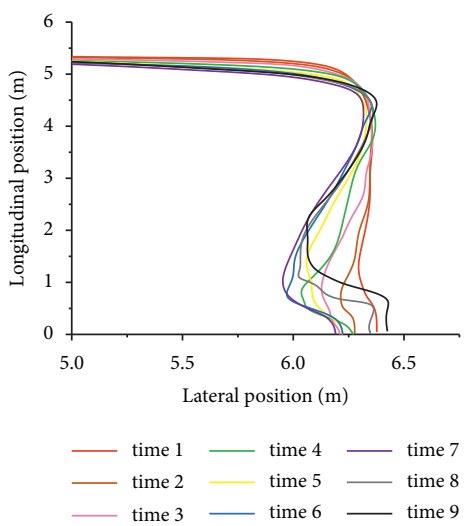

(a)
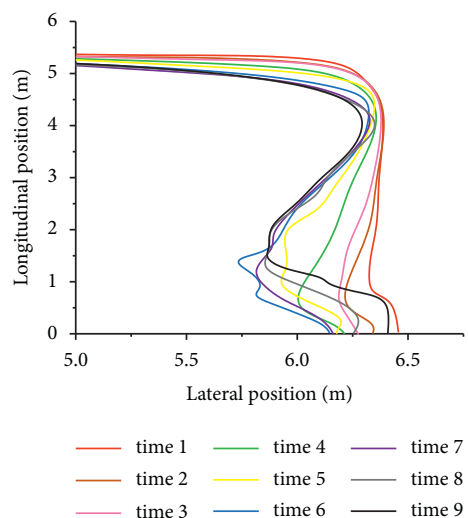

(d)
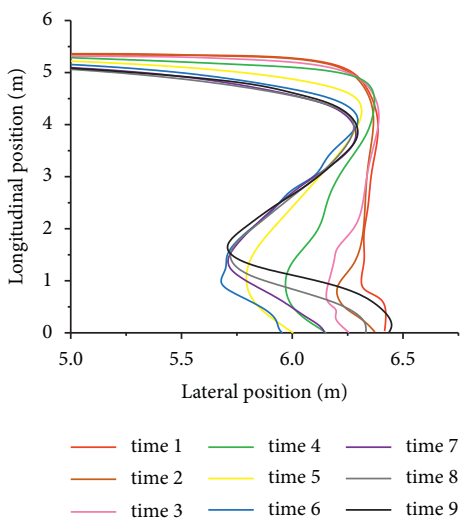

(g)

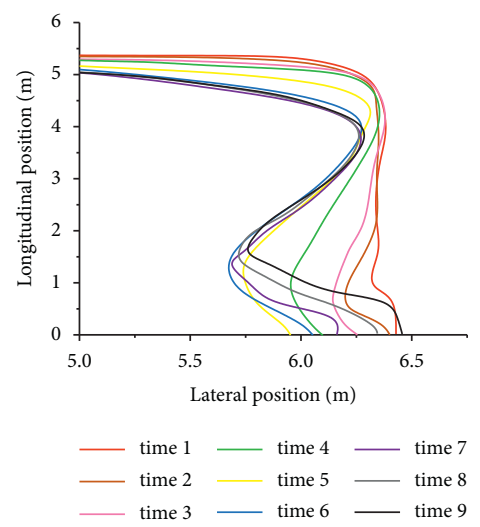

(j)
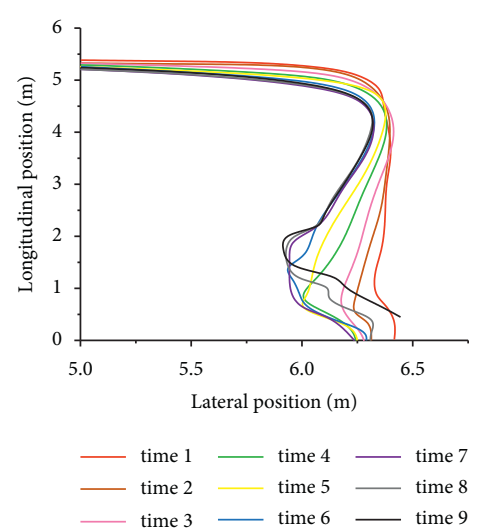

(b)
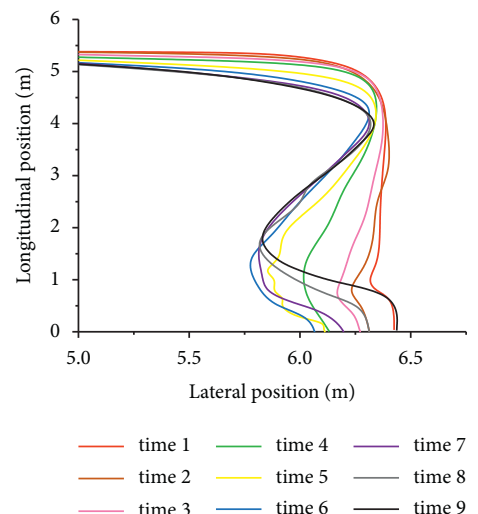

(e)
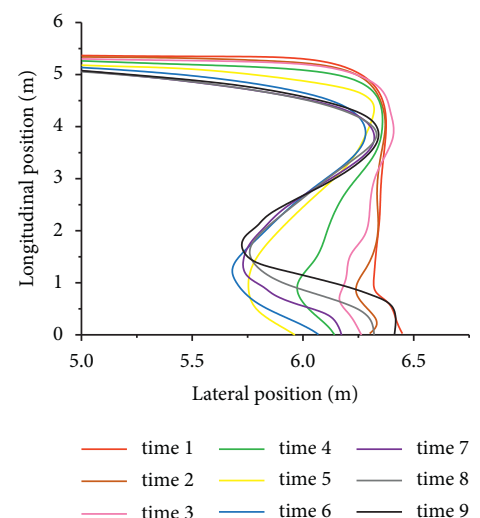

(h)
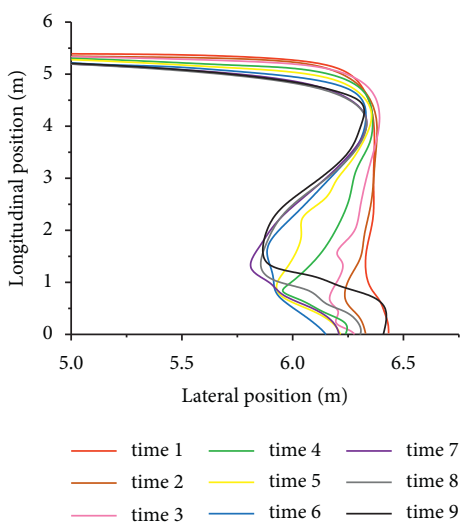

(c)
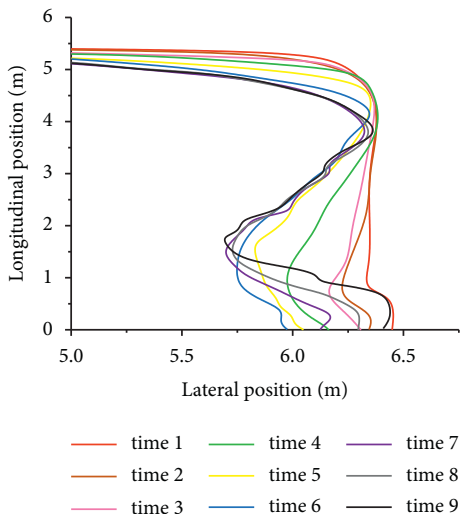

(f)
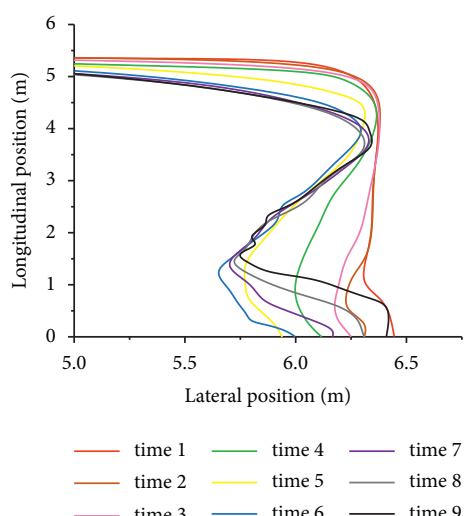

(i)

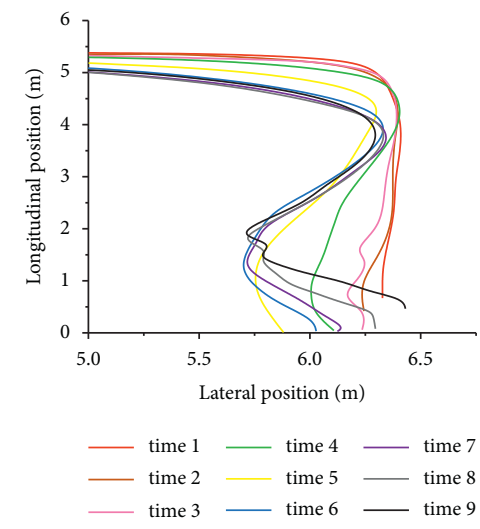

(k)

Figure 5: The coal gangue boundary curve at different times. (a) The boundary curve at $20^{\circ}$. (b) The boundary curve at $22^{\circ}$. (c) The boundary curve at $24^{\circ}$. (d) The boundary curve at $26^{\circ}$. (e) The boundary curve at $28^{\circ}$. (f) The boundary curve at $30^{\circ}$. (g) The boundary curve at $32^{\circ}$. (h) The boundary curve at $34^{\circ}$. (i) The boundary curve at $36^{\circ}$. (j) The boundary curve at $38^{\circ}$. (k) The boundary curve at $40^{\circ}$. 

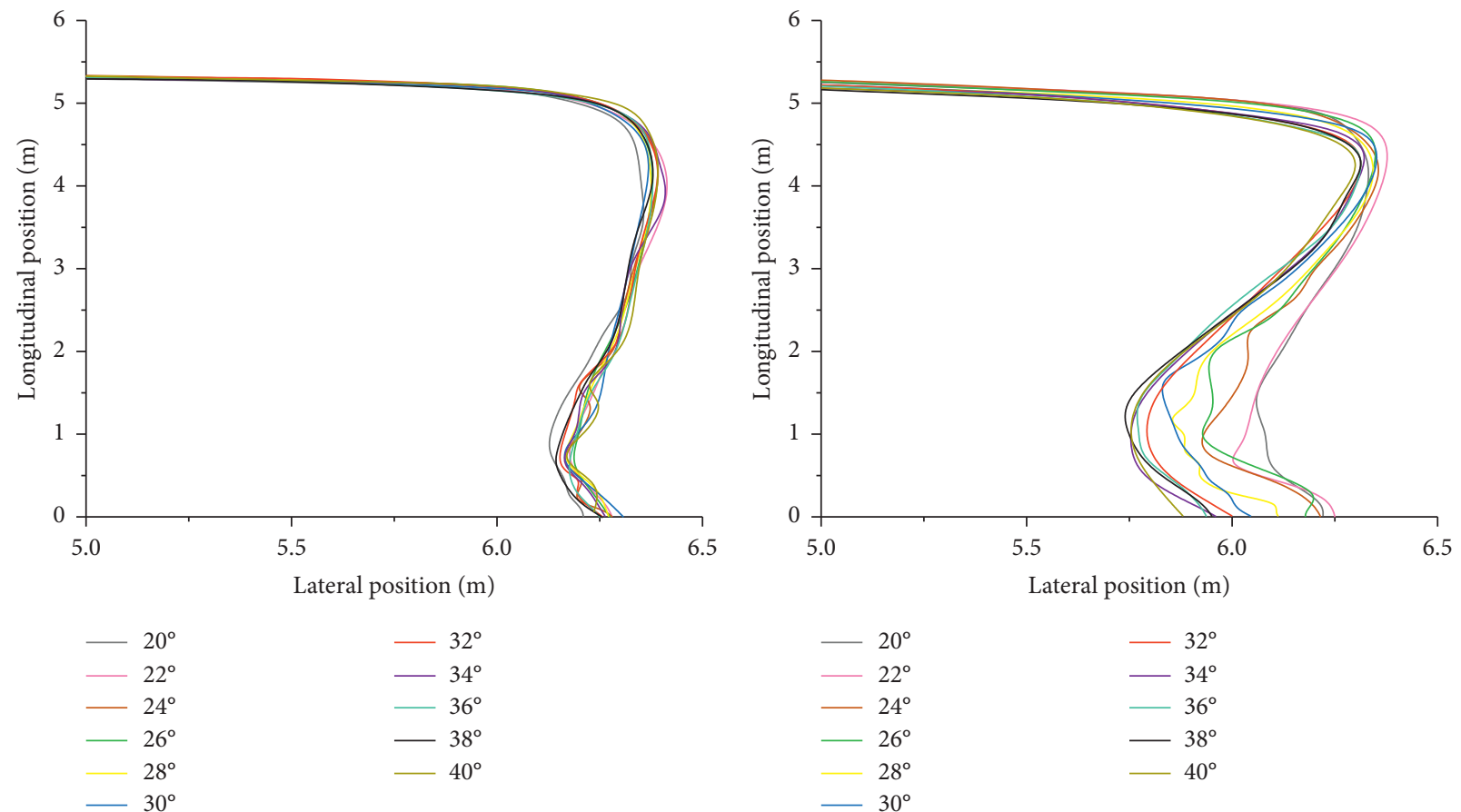

(a)

(b)
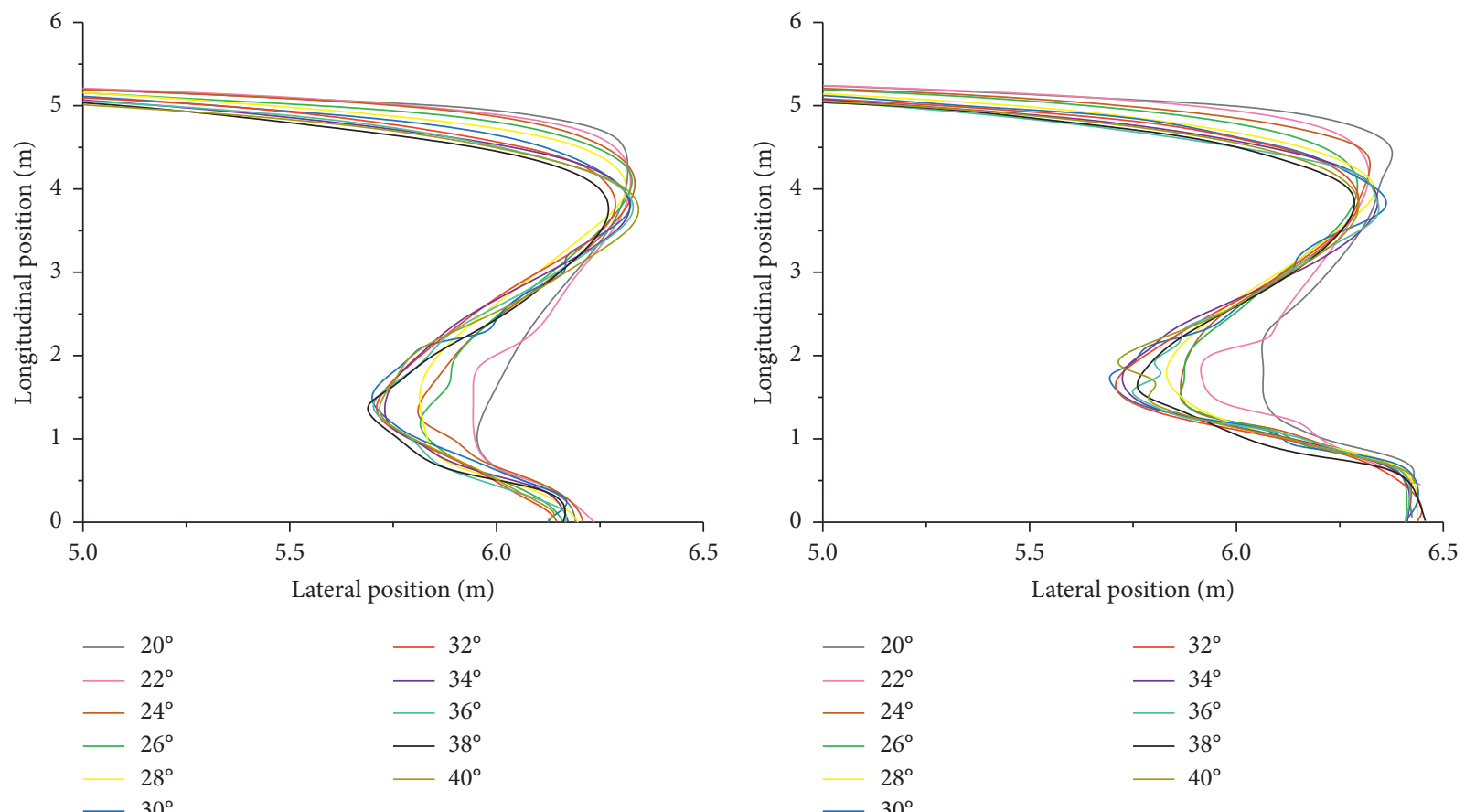

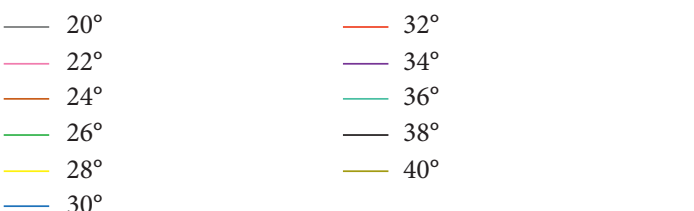

(c)

(d)

Figure 6: Comparison curve of the coal gangue boundary at different angles. (a) The boundary curve at $3 \mathrm{~s}$. (b) The boundary curve at $5 \mathrm{~s}$. (c) The boundary curve at $7 \mathrm{~s}$. (d) The boundary curve at $9 \mathrm{~s}$.

small, and the change rate is slow. When the coal caving time is $5 \mathrm{~s}$, the deformation extent of the coal gangue boundary is the smallest in the $20^{\circ}$ condition, while it is the largest in the $34^{\circ}$ to $40^{\circ}$ condition, but there is little difference between them. From $20^{\circ}$ to $32^{\circ}$, the influence of the tail beam swing angle on the change in the boundary is quite different. This shows that there is an apparent correlation between the tail beam swing angle and the coal gangue boundary. When the coal caving time is $7 \mathrm{~s}$, the deformation of the coal gangue boundary is the smallest in the $20^{\circ}$ condition, while it is the largest in the $30^{\circ}$ to $40^{\circ}$ condition, but there is little difference between them. From $20^{\circ}$ to $28^{\circ}$, the influence of the swing 
angle of the tail beam on the boundary change is quite different. When the coal caving time is $9 \mathrm{~s}$, the deformation at $20^{\circ}$ is small and relatively smooth, and the deformation at $22^{\circ}$ is small but large locally. Large deformation occurs between $24^{\circ}$ and $40^{\circ}$, irregular bending occurs in part, and there is more mixture of gangue particles near the coal gangue boundary.

From the comprehensive view of the 4 groups of curves in Figure 6, the tail beam swing at different angles has an evident influence on the change in the coal gangue boundary. Under the condition of small swing angles of the tail beam, such as $20^{\circ}$, $22^{\circ}$, and $24^{\circ}$, the coal gangue boundary is smoother, which indicates that the coal caving speed is uniform, and gangue is not easily mixed into the top coal. Under the condition of a significant swing angle of the tail beam, such as $30^{\circ}, 36^{\circ}$, and $40^{\circ}$, some irregular bending occurs on the coal gangue boundary, which means that coal gangue particles are mixed on the boundary, and gangue is easily mixed into the top coal. At all angles from $20^{\circ}$ to $40^{\circ}$, the coal gangue boundary showed that the upper part deformed downwards and the lower part deformed to the right and finally showed a double arch shape. In general, the upper part of the coal gangue boundary moves downward with increasing angle. This is because the larger the angle is, the larger the coal caving outlet is, and the faster the top coal caving speed is. The lower part of the boundary moves left with an increasing angle, and the left convex point of the curve is close to the middle line of the coal caving outlet. When the swing angle is less than $28^{\circ}$, the left displacement of the coal gangue boundary increases obviously with the swing angle; when the swing angle is more than $28^{\circ}$, the further left displacement of the coal gangue boundary changes slightly and only a small vibration. This phenomenon indicates that the limit position of the movement of the coal gangue boundary is near the middle line of the coal caving outlet and depends on the density difference of coal gangue particles. The greater the density difference between them is, the closer the boundary is to the middle line of the coal caving outlet.

Comparing Figures 5 and 6 comprehensively, under the same tail beam swing angle, with increasing caving time, the probability of gangue particles mixing into coal particles increases, and the coal gangue boundary gradually changes from smooth to complex. Under the same caving time, with the increase in the tail beam swing angle, the probability of gangue particles mixing into coal particles becomes larger, and the coal gangue boundary gradually changes from smooth to complex. Under all working conditions, the coal gangue boundary moves to the lower left. The upper part moves downward at a slower speed, while the lower part moves to the left faster and finally presents a double arch structure. When the tail beam swings down, the probability of gangue particles mixing with coal particles is very small. When the tail beam swings up, because the top coal receives the upward supporting force, the probability of mixing gangue and coal particles is large.

4.3. Influence of Caving Time and Angle on the Coal Gangue Discharge. To study the relationship between coal gangue discharge and caving time and angle, 9 time points are evenly selected in the same tail beam swing cycle, and the span of each time point is 1 second. The Grid Bin Group is added at the coal caving outlet of the simulation model, and the quantity and quality of coal and gangue particles passing through the Grid Bin Group are monitored and counted by the EDEM Selection Query Editor. Eleven groups of coal particle emissions and gangue particle emissions simulated from different angles are extracted, and a total of 22 groups of time-related data are obtained. Plotting the 9 time points on the $x$-axis, the 11 different angles on the $z$-axis, and the coal gangue discharge on the $y$-axis, two sets of $3 \mathrm{D}$ visualization curves are obtained. Each set includes 11 groups of curves, and each curve is associated with time points 1-9. These are shown in Figure 7.

According to the observation in Figure 7(a), the curve shape is roughly similar when the caving angle is different. In terms of quantity, they are rising continuously. In terms of speed, they accelerate first and then slow down gradually. The fastest coal caving speed is concentrated near time points 5-7. Comparing the curves of the 11 groups, it can be observed that there are some differences in curve shape with different caving angles. With the increasing caving angle, the coal discharge also increases. This shows that the larger the caving angle is, the higher the coal caving speed is. With the increasing caving angle, the interval between curves decreases. This shows that when the angle is small, the caving angle has a significant influence on the coal caving rate. When the angle is large, the caving angle has little effect on the coal caving rate. In addition, with increasing caving angle, the maximum slope point of the curve moves backward. This shows that the larger the coal caving outlet is, the longer the fully open time is, and the later the maximum coal caving rate point is. Similarly, from Figure 7(b), the shape of the gangue discharge curve is roughly similar when the caving angle is different. The discharge of gangue rises, and the discharge speed is accelerated first and then decelerated. The changes in other curve characteristics are also similar to the coal discharge curve. However, relative to the coal discharge curve, the velocity of the gangue curve changes more violently with time, and the corresponding maximum velocity of each curve is also greater. Because the density of gangue is much higher than that of coal, the caving time has a greater influence on the caving rate of gangue. The fastest curve speed of gangue discharge is concentrated near time points 6-7. Compared with the coal discharge curve, the slope of the gangue discharge curve changes faster. This means that the flow acceleration of gangue particles is larger, and the change time of the velocity peak is shorter. At the same time, it can be observed that the interval between gangue discharge curves is greater than that between coal discharge curves. This shows that the influence of the caving angle on gangue discharge is greater than that on coal discharge.

Generally, the caving characteristics of coal and gangue are the same: The discharge of coal gangue increases with time, and the increasing speed shows a trend of accelerating first and then slowing down. The speed of coal gangue discharge increases with increasing caving angle, and the increase is greater when the angle is small, while the increase is smaller when the angle is large. The caving characteristics 


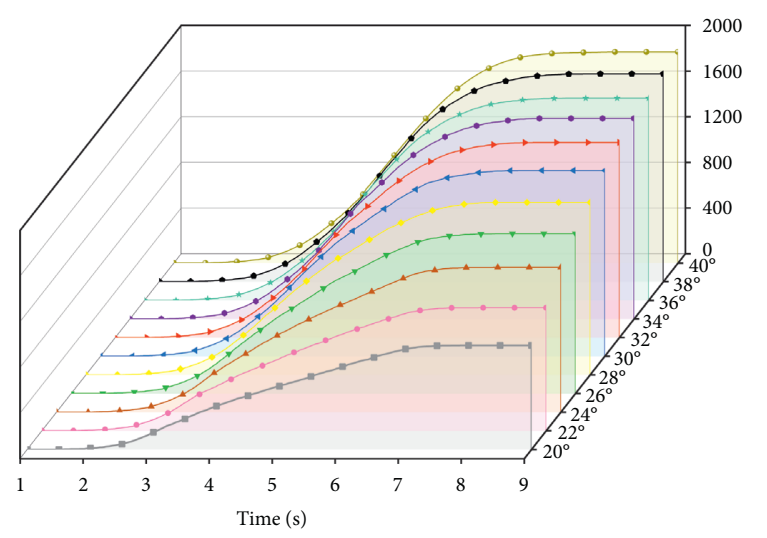

(a)

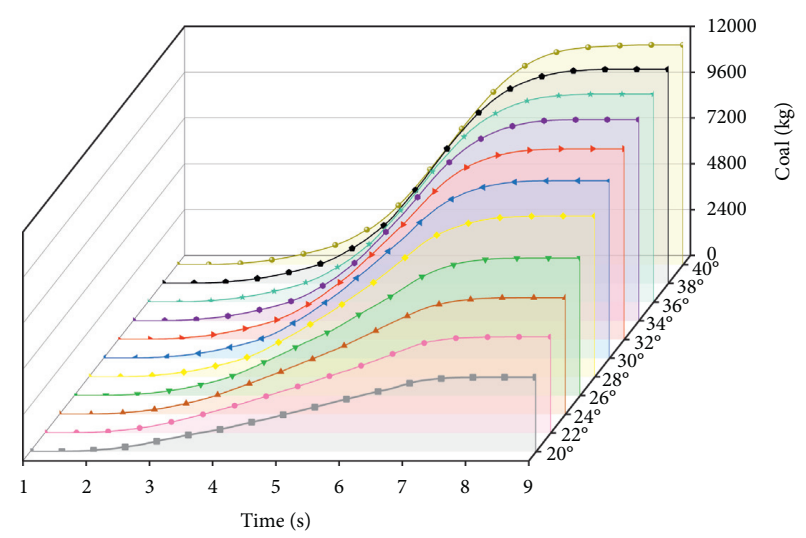

(b)

Figure 7: Variation trends of coal and gangue discharge. (a) Variation trend of coal discharge. (b) Variation trend of gangue discharge.

of coal and gangue are also different: Compared with coal, the discharge rate of gangue is more affected by caving time, and the flow acceleration of gangue particles is larger. Compared with coal, the discharge rate of gangue is more affected by the caving angle. An angle that is too large easily releases more gangue particles.

\section{Research Conclusion}

To study the influence of the tail beam swing angle of the caving hydraulic support and caving time on the evolution of the coal gangue boundary, the discrete element method was adopted. Based on Hertz-Mindlin (no-slip) contact theory and Hertz-Mindlin with bonding contact theory, a simulation model of the top coal caving process was established. Eleven groups of images of coal caving results under different conditions were extracted, the evolution data of the coal gangue boundary were extracted, and the evolution process of the coal gangue boundary was analyzed. In addition, the following five conclusions were obtained:

(1) The evolution of the coal gangue boundary has a limit position, which is near the middle line of the coal caving outlet. When the boundary reaches this position, the coal caving time and the tail beam swing angle will have a very weak influence.

(2) When the coal gangue boundary does not reach the limit position, the longer the caving time is, the greater the deformation of the boundary is. The gangue is close to the coal caving outlet, resulting in an increase in the gangue content in the top coal.

(3) When the coal gangue boundary does not reach the limit position, the larger the swing angle is, the faster the deformation rate of the boundary is, and the more prone the boundary is to irregular bending, resulting in the mixing of gangue with the top coal and an increase in gangue content.

(4) The evolution of the coal gangue boundary includes two directions: the upper part moves downwards and the lower part moves left, which finally makes the coal gangue boundary appear as a double arch structure.
(5) The increase in caving time and tail beam swing angle will increase the discharge of coal and gangue, but compared with coal, gangue is more affected by caving time and tail beam swing angle.

(6) The caving time and the tail beam swing angle significantly influence the movement of the coal gangue boundary. In engineering applications, it is necessary to select the appropriate caving time and tail beam swing angle by comprehensively considering the output, quality, and efficiency.

This paper provides a theoretical basis for the study of the evolution of the boundary. It is of great significance to improve the top coal caving yield, reduce the gangue content, improve the fully mechanized top coal caving mining technology, perfect the mining theory system, and accelerate the construction of smart mines.

\section{Data Availability}

The data used to support the findings of this study are included within the article.

\section{Additional Points}

(1) The evolutionary mechanism of the boundary between coal and gangue in top coal caving is studied. (2) The influence of the tail beam swing angle and coal caving time on the evolution of the coal gangue boundary is innovatively studied. (3) The evolution curve of coal and gangue is drawn. (4) The influence of different evolution forms of the coal gangue boundary on the final caving effect is analyzed.

\section{Disclosure}

Shandong University of science and technology and Shandong Normal University contributed to the work equally and should be regarded as co-first units.

\section{Conflicts of Interest}

The authors declare that they have no conflicts of interest. 


\section{Acknowledgments}

This work was supported by the National Natural Science Fund of China (Grant no. 51974170) and Special funds for the Climbing Project of Taishan Scholars.

\section{References}

[1] J. Wang, "Development and prospect on fully mechanized mining in Chinese coal mines," International Journal of Coal Science \& Technology, vol. 1, no. 3, pp. 253-260, 2014.

[2] N. Zhang, C. Liu, and P. Yang, "Flow of top coal and roof rock and loss of top coal in fully mechanized top coal caving mining of extra thick coal seams," Arabian Journal of Geosciences, vol. 9, no. 6, pp. 1-9, 2016.

[3] Z. Y. Song and J. W. Zhang, "Numerical simulation of topcoal thickness effect on the top-coal recovery ratio by using DEM method," Electronic Journal of Geotechnical Engineering, vol. 20, no. 9, pp. 3795-3807, 2015.

[4] J. C. Wang, Y. Chen, and J. W. Zhang, "Optimization study on drawing technique of longwall top-coal caving in extra-thick coal seam based on BBR system," COAL ENGINEERING, vol. 48, no. 2, pp. 1-4, 2016.

[5] D. Szurgacz and J. Brodny, "Tests of geometry of the powered roof support section," Energies, vol. 12, no. 20, p. 3945, 2019.

[6] Q. Zhang, J. -x. Zhang, X. -l. Han, F. Ju, Y. Tai, and M. Li, "Theoretical research on mass ratio in solid backfill coal mining," Environmental Earth Sciences, vol. 75, no. 7, p. 586, 2016.

[7] Z. Zhang, "Fracture development law of overlying strata in fully mechanized caving mining," Industrial Safety and Environmental Protection, vol. 45, no. 5, pp. 14-16+22, 2019.

[8] B. Unver and N. E. Yasitli, "Modelling of strata movement with a special reference to caving mechanism in thick seam coal mining," International Journal of Coal Geology, vol. 66, no. 4, pp. 227-252, 2006.

[9] Z. W. Zhang and J. N. Wu, "Full-mechanized caving mining technology of extremely thick and hard coal-seam with hard roof in shallow buried depth," Strategic Study of CAE, vol. 13, no. 11, pp. 107-112, 2011.

[10] J. Hu, M. He, J. Wang, Z. Ma, Y. Wang, and X. Zhang, "Key parameters of roof cutting of gob-side entry retaining in a deep inclined thick coal seam with hard roof," Energies, vol. 12 , no. 5 , p. $934,2019$.

[11] N. B. Zhang, C. Y. Liu, and Y. M. Chen, "Study on coal and gangue flow rule of top-coal caving with erratic hick coal seam by PFC2D," Coal Technology, vol. 33, no. 12, pp. 1-4, 2014.

[12] D. Szurgacz and J. Brodny, "Adapting the powered roof support to diverse mining and geological conditions," Energies, vol. 13, no. 2, p. 405, 2020.

[13] C. Y. Liu, B. X. Huang, P. J. Yang, and P. F. Wu, "Law of Coal Gangue Flow Field and Determination of Coal Caving Process Parameters in Fully Mechanized Top Coal Caving Face with Large Mining Height," Innovative development of theory and practice of fully mechanized top coal caving technology, Vol. 7, Institute of mining technology, mining design division of Tiandi science and Technology Co, Ltd. (mining design and research branch of General Coal Research Institute), , Beijing, China, 2012.

[14] B. Yu, D. J. Zhu, and Z. H. Chen, "Top-coal drawing law of LTCC mining based on stochastic medium theory," Journal of China Coal Society, vol. 42, no. 6, pp. 1366-1371, 2017.

[15] D. J. Zhu, Z. H. Chen, Y. Chang, and Z. H. Zhou, "Study on top coal caving law of fully-mechanized top coal caving mining based on random medium theory," Coal Science and Technology, vol. 46, no. 1, pp. 167-174, 2018.

[16] J. W. Zhang, Simulation Study on the Three-Dimensional Caving Mechanism of Loose Top-Coal in Longwall Top-Coal Caving Mining Panel, China University of mining and Technology, Beijing, China, 2017.

[17] S. X. Zhang, X. L. Zhang, S. Liu, and G. Q. Xu, "Intelligent precise control technology of fully mechanized top coal caving face," Journal of China Coal Society, vol. 45, no. 6, pp. 2008-2020, 2020.

[18] H. Jia, L. Wang, K. Fan, B. Peng, and K. Pan, "Control technology of soft rock floor in mining roadway with coal pillar protection: a case study," Energies, vol. 12, no. 15, p. 3009, 2019.

[19] J. Dai, P. Shan, and Q. Zhou, "Study on intelligent identification method of coal pillar stability in fully mechanized caving face of thick coal seam," Energies, vol. 13, no. 2, pp. 305-317, 2020.

[20] X. He, C. Y. Liu, and F. F. Wu, "Effect of elevation angle of coal seam on top coal flow and release in fully mechanized caving face during topple mining," Coal Science and Technology, vol. 49, no. 9, pp. 25-31, 2021.

[21] S. L. Yang, B. Zhao, and L. H. Li, "Coal wall failure mechanism of longwall working face with false dip in steep coal seam," Journal of China Coal Society, vol. 44, no. 2, pp. 367-376, 2019.

[22] Y. Lan, R. Gao, B. Yu, and X. Meng, "In situ studies on the characteristics of strata structures and behaviors in mining of a thick coal seam with hard roofs," Energies, vol. 11, no. 9, p. 2470, 2018.

[23] X. Liu, D. Fan, Y. Tan et al., "Failure evolution and instability mechanism of surrounding rock for close-distance parallel chambers with super-large section in deep coal mines," International Journal of Geomechanics, vol. 21, no. 5, Article ID 04021049, 2021.

[24] C. Kloss, C. Goniva, A. Hager, S. Amberger, and S. Pirker, "Models, algorithms and validation for opensource DEM and CFD-DEM," Progress in Computational Fluid Dynamics, An International Journal, vol. 12, no. 2/3, pp. 140-152, 2012.

[25] X. Liu, D. Fan, Y. Tan, and J. Ning, "New Detecting Method on the Connecting Fractured Zone above the Coal Face and a Case Study," Rock Mech Rock Eng, vol. 54, no. 1-2, pp. 1-13, 2021.

[26] C. O'Sullivan, "Particle-based discrete element modeling: geomechanics perspective," International Journal of Geomechanics, vol. 11, no. 6, pp. 449-464, 2011.

[27] S. Utili, T. Zhao, and G. T. Houlsby, "3D DEM investigation of granular column collapse: evaluation of debris motion and its destructive power," Engineering Geology, vol. 186, pp. 3-16, 2015.

[28] H. Hertz, "On the contact of elastic solids," Journal für die rne und angewandte Mathematik, Crelles Journal, vol. 92, no. 156, 1880.

[29] R. D. Mindlin and H. Deresiewicz, "Elastic spheres in contact under varying oblique forces," Journal of Applied Mechanics, vol. 20, no. 3, pp. 327-344, 1953.

[30] G. Q. Wang, W. J. He, and J. X. Wang, Discrete Element Method and its Application in EDEM, Northwest University of Technology Press, Xi'an, China, 2010.

[31] M. Liu and Z. P. Liu, "Analysis of the main factors affecting the accuracy of bituminous coal caking index," 2014 China coal preparation development forum, Vol. 7, China coal processing and Utilization Association, , Beijing, China, 2014.

[32] X. Liu, S. Song, Y. Tan et al., "Similar simulation study on the deformation and failure of surrounding rock of a large section 
chamber group under dynamic loading," International Journal of Mining Science and Technology, vol. 31, no. 3, pp. 495-505, 2021.

[33] S. Zhong, Simulation Analysis of the Coal Releasing Process Characteristics and the Coupling Characteristics between the ZF15000/28/52 Top-Coal Caving Hydraulic Support and the Surrounding Rocks, Liaoning University of engineering and technology, Liaoning, China, 2017.

[34] T. J. Li, Calibration of Dem Model Parameters for Coal Particles and Research on Relationships between Macro and Micro Parameters, Taiyuan University of Technology, Taiyuan, China, 2019. 